\title{
Management of primary squamous cell carcinoma of the pancreas with a nanosomal paclitaxel lipid suspension-based regimen: A case report
}

\author{
SAROJ KUMAR DAS MAJUMDAR ${ }^{1}$, DILLIP KUMAR MUDULY ${ }^{2}$, SUBHASIS MISHRA ${ }^{2}$, \\ CHINMAYA RASHMI RANJAN MOHAPATRA ${ }^{2}$, DEEPAK BUNGER ${ }^{3}$ and MUJTABAA. KHAN ${ }^{3}$ \\ Departments of ${ }^{1}$ Radiotherapy and ${ }^{2}$ Surgical Oncology, All India Institute of Medical Sciences, \\ Bhubaneswar, Odisha 751019; ${ }^{3}$ Department of Medical Affairs and Clinical Development, \\ Intas Pharmaceuticals Ltd., Ahmedabad, Gujarat 380054, India
}

Received October 22, 2018; Accepted February 1, 2019

DOI: $10.3892 /$ mco.2019.1814

\begin{abstract}
Squamous cell carcinoma (SCC) of the pancreas is a rare tumor with only a few case reports available. It is an aggressive form of pancreatic cancer with a poor prognosis. The diagnosis and optimal management of SCC of the pancreas is poorly defined due to the lack of standard treatment or guidelines and owing to the rarity of this malignancy. Patients suffering from SCC of the pancreas do not respond well to chemotherapy or radiotherapy and isolated reports are available on the use of gemcitabine and newer taxane formulations. Surgical resection of the tumor is the most effective modality; however, due to a delay in diagnosis, the majority of pancreatic SCCs remain unresectable. Herein, a case of SCC of the pancreatic tail is reported in a 60-year-old patient who was managed with a combination of albumin-free nanosomal paclitaxel lipid suspension (NPLS) and gemcitabine in a neoadjuvant setting. To the best of our knowledge, this is the first such case report of a locally advanced SCC of the pancreatic tail showing an overall survival of 1 year following treatment with an NPLS based regimen. The treatment was well tolerated with no serious safety concerns.
\end{abstract}

\section{Introduction}

Pancreatic cancer is a highly lethal malignancy with short-term survival rates (1) and is associated with a high mortality rate that closely parallels its incidence (2). It is generally considered as a 'silent disease' (3) as there are no signs and symptoms

Correspondence to: Dr Saroj Kumar Das Majumdar, Department of Radiotherapy, All India Institute of Medical Sciences, Bhubaneswar, Odisha 751019, India

E-mail: sarojmajumdar@gmail.com

Key words: pancreatic cancer, PacliAqualip, nanosomal paclitaxel lipid suspension, pancreatic tail, squamous cell carcinoma in its early stages, and is generally diagnosed at an advanced stage $(2,3)$. The number of new cases of pancreatic cancer were 12.6 per 100,000 men and women per year according to the National Cancer Institute Surveillance, Epidemiology, and End Results Program (NCI SEER) data from 2011-2015 (4). In India, the incidence of pancreatic cancer is comparatively low at 0.5-2.4 per 100,000 men and 0.2-1.8 per 100,000 women (5). Squamous cell carcinoma (SCC) of pancreas is a subtype of ductal carcinoma, a non-endocrine part (6). Primary pancreatic SCC is a rare malignancy, accounting for $0.5-2 \%$ of all malignant pancreatic tumors (7) and is considered as an aggressive subtype with a poor prognosis (8). The diagnosis and optimal management of SCC of pancreas remains poorly defined (9). Furthermore, there is no standard treatment or guidelines available for the management of SCC of pancreas owing to the rarity of this malignancy $(10,11)$.

We report here, a case of locally advanced SCC of the pancreatic tail. The patient was treated with neoadjuvant nanosomal paclitaxel lipid suspension (NPLS) and gemcitabine chemotherapy followed by surgery.

\section{Case report}

A 60-year-old man, a known case of diabetes, presented at the Department of Oncology, All India Institute of Medical Sciences (AIIMS), Bhubaneswar, Odisha, India, in February 2017, with complaints of abdominal pain (epigastric pain radiating to back), vomiting, weight loss (10 kg over the last 6 months) and weakness. He was a non-smoker and non-alcoholic. Investigations revealed that his baseline serum amylase (17.49 IU/l), lipase (16.76 U/l) and CA 19-9 (29.9 U/ml) levels were within normal limits. An upper gastrointestinal endoscopy (UGIE) showed a submucosal swelling which measured 10x10 cm. A computed tomography (CT) scan of the abdomen revealed a heterogeneously enhancing cystic and necrotic mass lesion, measuring $8.1 \times 6.8 \times 6.4 \mathrm{~cm}$ in the pancreatic tail, with chronic calcific pancreatitis, diffuse parenchymal calcification and multiple studded intraductal calculi; the pancreatic tail region was lobulated near the hilum with splenic vessel encasement, and a secondary splenic 


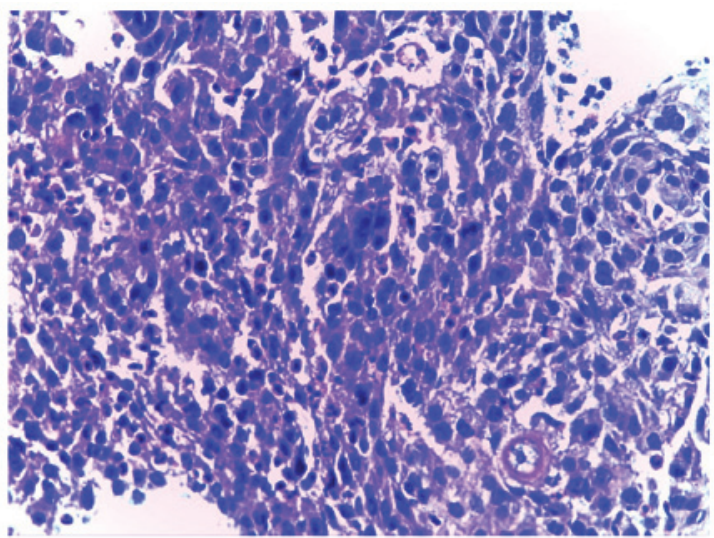

Figure 1. Markedly pleomorphic tumor cells in diffuse sheets (magnification, x200).

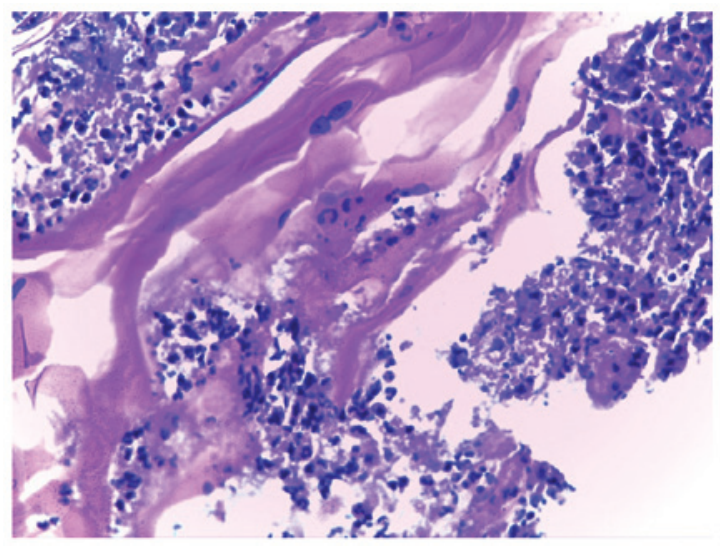

Figure 2. Large areas of keratinisation and necrosis (magnification, $\mathrm{x} 400$ ).

parenchymal infarct (images not available). A CT guided core needle biopsy of the pancreatic mass was performed, and histologic examination revealed markedly pleomorphic tumor cells in diffuse sheets (Fig. 1) and large areas of keratinization and necrosis (Fig. 2). The patient was diagnosed as a case of primary SCC of pancreas, a rare entity, which arises from the pancreatic duct in the background of chronic calcific pancreatitis or a pre-existing pancreatic stone. The staging was confirmed as Stage III (T4N1M0). Extensive workup performed to evaluate other primary sources and metastatic disease did not reveal any head and neck malignancy. The final diagnosis was confirmed as primary SCC of pancreatic tail.

The Eastern Cooperative Oncology Group (ECOG) performance status was 1 , and neoadjuvant chemotherapy with a combination of NPLS $120 \mathrm{mg}\left(70 \mathrm{mg} / \mathrm{m}^{2}\right)$ and gemcitabine $1400 \mathrm{mg}\left(800 \mathrm{mg} / \mathrm{m}^{2}\right)$ intravenously (IV) was initiated on days 1,8 and 15 every 4 weeks. Premedication with IV ranitidine $(50 \mathrm{mg})$, dexamethasone $(8 \mathrm{mg})$, chlorpheniramine maleate $(10 \mathrm{mg})$, and ondansetron $(8 \mathrm{mg})$ was given. On day 1 of the 1 st cycle, NPLS $120 \mathrm{mg}$ was reconstituted in $250 \mathrm{ml}$ of $5 \%$ dextrose solution and was administered over $1.5 \mathrm{~h}$; gemcitabine 1.4 was reconstituted in $250 \mathrm{ml}$ normal saline and was administered over $45 \mathrm{~min}$. The patient complained of nausea and was administered ranitidine $50 \mathrm{mg}$ IV and ondansetron $8 \mathrm{mg}$ IV. The patient received the same premedications and chemotherapy on days 8 and

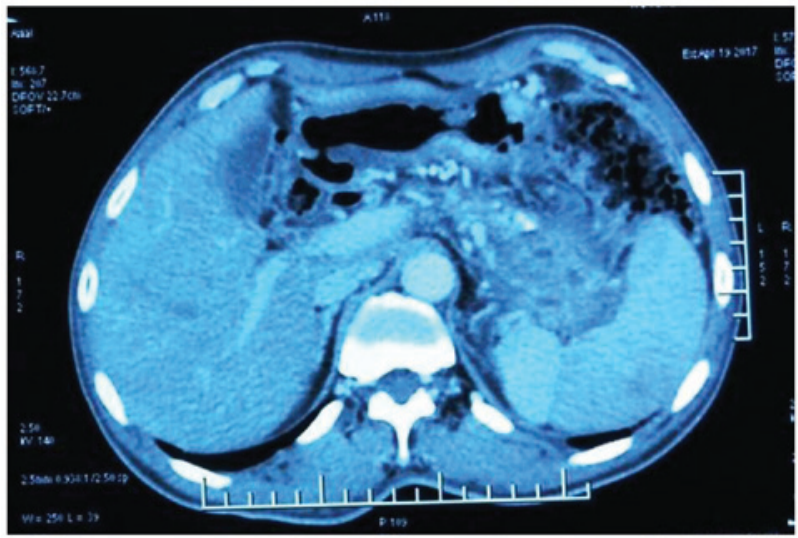

Figure 3. Positron emission tomography-computed tomography image following 2 cycles of chemotherapy, showing a $2.3 \times 1.5 \times 2.1 \mathrm{~cm}$ mass in the tail of pancreas.

15 of Cycle 1. The patient tolerated the treatment well; vital signs, liver and renal functions were normal, and the patient was hemodynamically stable. Similarly, the second cycle of NPLS-gemcitabine chemotherapy was administered on days 1,8 and 15 . On day 1 of the second cycle, the patient's random blood sugar (RBS) level was found to be high (517 mg/dl), which was managed with insulin. On day 15 of the second cycle, the patient's hemoglobin $(\mathrm{Hb})$ levels became low $(8.6 \mathrm{gm} \%)$ and he was administered 1 unit of packed red blood cells.

Post completion of 2 cycles of chemotherapy, a positron emission tomography-CT (PET CT) performed in April 2017 showed hypodense areas in the region of the splenic hilum and pancreatic tail, along with diffuse peritoneal stranding $(2.3 \times 1.5 \times 2.1 \mathrm{~cm})$, and with standardized uptake values (SUV) of 4.2, suggestive of a good tumoral response with the regression of tumor mass (Fig. 3). There was no hypermetabolic abdominal or retroperitoneal lymphadenopathy, and no definite abnormal hypermetabolic distant metastases were observed. The treatment was well-tolerated, and the patient received a third cycle of NPLS-gemcitabine combination therapy in May 2017 without any new complaints.

Post 3 cycles of chemotherapy, the patient was asymptomatic and tolerated the treatment with NPLS and gemcitabine well with normal complete blood counts (CBC), liver and renal function tests. Peripheral neuropathy (grade II) and anemia (grade III) were the adverse events reported in this patient.

Based on the tumoral response on the PET-CT, the patient was offered surgical exploration. He underwent distal pancreatico-splenectomy with enblock resection of the involved stomach and colon in June 2017 and R0 resection was achieved. Biopsy specimens from the pancreatic margin, celiac lymph node and right common hepatic lymph node confirmed a well-differentiated SCC of the tail of pancreas infiltrating into the wall of stomach and colon. The staging was confirmed as Stage IIA (pT3NOMx) within the limitations of metastatic work-up, and the patient was discharged in stable condition.

Post-surgery, the patient received 2 cycles of gemcitabine $1 \mathrm{gm}$ IV (day 1, 8 and 15 of each cycle) in combination with capecitabine $1,000 \mathrm{mg}$ orally (day 1 to 21 ) as adjuvant therapy. The patient became asymptomatic and tolerated the treatment well. 
Outcome and follow-up. Seven months post completion of NPLS and gemcitabine therapy, an ultrasound (USG) performed in December 2017 revealed multiple metastatic lesions in the liver, a segment 5 lesion causing infiltration of the common bile duct and resultant bilobar intrahepatic biliary radical dilatation (IHBRD) and minimal ascites. The patient underwent percutaneous transhepatic biliary drainage (PTBD) with stenting, but succumbed after 2 months, leading to an overall survival (OS) of approximately 1 year after having started NPLS treatment.

\section{Discussion}

Pancreatic cancer is one of the deadliest malignancies, and is associated with a poor prognosis (12). The symptoms can vary depending upon the anatomic location of the tumor. The head of the pancreas (78\%) is most commonly affected, followed by the body (11\%) and tail (11\%) (3). Cancers of the pancreatic head are located near the common bile duct, may cause jaundice even if they are small, and can be diagnosed at an early stage. However, cancers of the pancreatic body or tail don't impinge on the duct until they have spread through the pancreas and are at an advanced stage (13). The survival rates are also lower in patients with pancreatic cancers of the body and tail as compared with head lesions (14). Primary SCC of pancreas is an extremely rare entity, with an incidence of 0.5 to $2 \%$ of all exocrine pancreatic neoplasms (15). The clinical presentation of SCC of pancreas is similar to that of adenocarcinoma; it may arise from chronic inflammation leading to malignant transformation of squamous metaplasia, or from mixed adenosquamous carcinoma in which the glandular components have disappeared, or from an adenocarcinoma with squamous metaplasia where the tumor has a potential to drift into both adenocarcinoma or SCC (16). The correct diagnosis of SCC of pancreas requires a thorough workup to exclude another possible primary metastatic SCC (10).

There is no standard therapy established for the management of pancreatic SCC (10). These tumors generally do not respond to chemotherapy or radiotherapy and are very aggressive. Majority of the tumors may not be resectable due to a delay in diagnosis (15). Published literature is available only as case reports, and comprehensive clinical trials are not available (17).

The cure rate for pancreatic cancer is very low at $7 \%$, which makes the disease a vexing treatment challenge (18). Surgical resection is regarded as the only potentially curative treatment and Stage I, IIA and IIB pancreatic cancers are considered resectable (19). Neoadjuvant chemotherapy followed by surgical exploration has been studied in patients with locally advanced pancreatic cancer (20). Gemcitabine is considered as the standard of care despite its limited activity and modest treatment outcomes (12). It was shown to be effective in a 49-year-old patient with a metastatic SCC of the pancreas. Given as a second line therapy after radiotherapy plus $5 \mathrm{FU}$ and cisplatin, gemcitabine improved the patient's health leading to an OS of 8 months (21). In an inoperable patient, first-line therapy with gemcitabine and cisplatin followed by second-line 5-FU and leucovorin showed a good survival rate (22). Reports have shown improved outcomes in patients with pancreatic cancer with a taxane and gemcitabine combination $(12,18)$. The novel formulations of taxanes penetrate tumor cell mass at higher concentrations due to their unique structure and may exert better activity than conventional taxanes (12). Nab-paclitaxel, an albumin bound formulation of paclitaxel, has been studied in pancreatic adenocarcinoma $(23,24)$ but no data is available on its use in SCC. Kataoka et al demonstrated that nab-paclitaxel plus gemcitabine combination therapy was effective in a 60-year-old patient with adenosquamous carcinoma of the pancreas. However, disease progression was noted after the completion of 4 cycles, with an OS of 7 months (25).

Previous reports have shown a median OS of 7 months in 8 patients with pancreatic SCC undergoing curative resection $(26,27)$. Brown et al (28) demonstrated a median OS of 7 months (range 6-16 months) for patients who underwent curative resection. A recent systematic review and pooled survival analysis conducted by Ntanasis-Stathopoulos et al showed a median OS of 7 months in 54 patients with pancreatic SCC. This is the largest pool of data regarding pancreatic SCC available in public domain till date (29). The median age of patients at diagnosis was 63 years (range 33-80 years); majority $(61.1 \%)$ of them were males; pain $(77.8 \%)$ and weight loss $(57.4 \%)$ were the most common presenting symptoms. Most of the patients were nonsmokers (77.8\%) and most did not consume alcohol (70.4\%). Majority of the tumors were in the head of the pancreas $(52.9 \%)$ followed by tail $(21.6 \%)$ and body (5.9\%). Resectable cases had significantly better OS when compared with non-resectable cases (10 months versus 4 months, respectively) (29). OS without any treatment has been dismal and has been reported as 3 months (22). Furthermore, there were 11 cases of SCC of pancreatic tail in the pooled analysis, and the OS ranged from 2 to 8 months. In comparison, our patient initially presented with abdominal pain and weight loss, was a non-smoker and non-alcoholic and showed a higher OS of 1 year. This is the first case report on the use of NPLS in the treatment of primary SCC of pancreatic tail. In the current report, the patient had a locally advanced SCC of the pancreatic tail and was treated with an NPLS and gemcitabine combination therapy followed by surgery, thereby achieving a partial response. Overall, the treatment was well-tolerated, and no serious adverse events (SAEs) were reported. Neuropathy and anemia were observed, which resolved with appropriate treatment.

PacliAqualip is an albumin-free NPLS formulation, which is made from Lipids Generally Regarded As Safe (GRAS) by the US Food and Drug Administration (30). NPLS has been formulated based on 'Aqualip Technology', which is patented in Europe, Japan and Canada, while the filed US patent is under review. The Aqualip technology results in the drug particles being nano-sized $(\sim 100 \mathrm{~nm})$ that allows the drug to penetrate the tumor tissue through leaky vasculature without being attacked by the immune system. NPLS is devoid of cremophor EL and ethanol, thus potentially reducing the toxicities associated with cremophor for example severe anaphylactoid hypersensitivity reactions, hyperlipidemia, abnormal lipoprotein patterns, aggregation of erythrocytes and peripheral neuropathy (31). Furthermore, corticosteroid premedication is also not 
required. NPLS has been shown to be effective in the treatment of metastatic breast cancer (30). It is approved for the treatment of metastatic or node-positive breast cancer, metastatic ovarian cancer, metastatic non-small cell lung cancer, and AIDS-related Kaposi's sarcoma.

In our case, the patient with locally advanced SCC of pancreatic tail was treated with a novel nanosomal formulation of paclitaxel, NPLS, in combination with gemcitabine in a neoadjuvant setting followed by surgery. Overall, the current report highlights the potential efficacy and safety of NPLS in the treatment of SCC of pancreatic tail.

Presently, there is no definitive treatment available for squamous cell carcinoma of pancreas. This case report shows that NPLS, a novel albumin-free nanosomal paclitaxel formulation devoid of cremophor EL and ethanol, can be a potential treatment option for the treatment of locally advanced squamous cell carcinoma of pancreatic tail.

\section{Acknowledgements}

The authors would like to thank Mr. Shreekant Sharma (Lambda Therapeutic Research, Gujarat, India) for his support in developing the concept/medical writing, and follow-up with the journal/publisher, and Dr Venugopal Madhusudhana (Lambda Therapeutic Research) for the additional editorial assistance.

\section{Funding}

The publication of this case report was supported by a research grant from Intas Pharmaceuticals Ltd.

\section{Availability of data and materials}

All data generated or analyzed during this study are included in this published article.

\section{Authors' contributions}

SKDM, DKM, SM and CRRM planned and conducted the study, performed the procedures, acquired the data and wrote the manuscript. SKDM, DKM, SM, CRRM, DB and MAK conceived and designed the case report. SKDM, DKM, SM, CRRM, DB and MAK reviewed the manuscript, and SKDM served as the guarantor.

\section{Ethics approval and consent to participate}

Not applicable.

\section{Patient consent for publication}

Written informed consent was obtained from the patient's next of kin for the publication of this report as the patient was deceased.

\section{Competing interests}

Drs. Deepak Bunger and Mujtaba A. Khan are employees of Intas Pharmaceuticals Ltd.

\section{References}

1. Pourhoseingholi MA, Ashtari S, Hajizadeh N, Fazeli Z and Zali MR: Systematic review of pancreatic cancer epidemiology in Asia-Pacific Region: Major patterns in GLOBACON 2012. Gastroenterol Hepatol Bed Bench 10: 245-257, 2017.

2. Kamisawa T, Wood LD, Itoi T and Takaori K: Pancreatic cancer. Lancet 388: 73-85, 2016.

3. Lin HL, Kuo LC, Chen CW, Lin YK and Lee WC: Pancreatic tail cancer with sole manifestation of left flank pain: A very rare presentation. Kaohsiung J Med Sci 24: 324-327, 2008.

4. National Cancer Institute: Surveillance, epidemiology, and end results program. Cancer stat facts: Pancreatic cancer. https://seer.cancer.gov/statfacts/html/pancreas.html. Accessed June 29, 2018.

5. Dhir V and Mohandas KM: Epidemiology of digestive tract cancers in India IV. Gall bladder and pancreas. Indian J Gastroenterol 18: 24-28, 1999.

6. Al-Shehri A, Silverman S and King KM: Squamous cell carcinoma of the pancreas. Curr Oncol 15: 293-297, 2008.

7. Schultheis AM, Nguyen GP, Ortmann M, Kruis W, Büttner R, Schildhaus HU and Markiefka B: Squamous cell carcinoma of the pancreas in a patient with germline BRCA2 mutation-response to neoadjuvant radiochemotherapy. Case Rep Oncol Med 2014: 860532, 2014.

8. Makarova-Rusher OV, Ulahannan S, Greten TF and Duffy A: Pancreatic squamous cell carcinoma: A population-based study of epidemiology, clinicopathologic characteristics and outcomes. Pancreas 45: 1432-1437, 2016.

9. Kodavatiganti R, Campbell F, Hashmi A and Gollins SW: Primary squamous cell carcinoma of the pancreas: A case report and review of the literature. J Med Case Reports 6: 295-295, 2012.

10. Mehta M, Sinha J, Ogawa M, Ganguly A, Xiang D and Poddar N: Unusual Case of Squamous Cell Carcinoma of Pancreas with Review of Literature. J Gastrointest Cancer 46: 426-429, 2015.

11. NCCN Clinical Practice Guidelines in Oncology: Pancreatic Adenocarcinoma. V1. 2016. https://www.nccn.org/professionals/ physician_gls/pdf/pancreatic.pdf. Accessed June 30, 2018.

12. Belli C, Cereda S and Reni M: Role of taxanes in pancreatic cancer. World J Gastroenterol 18: 4457-4465, 2012.

13. American Cancer Society: Signs and symptoms of pancreatic cancer. https://www.cancer.org/cancer/pancreatic-cancer/ detection-diagnosis-staging/signs-and-symptoms.html. Accessed 28 February, 2018.

14. Artinyan A, Soriano PA, Prendergast C, Low T, Ellenhorn JD and Kim J: The anatomic location of pancreatic cancer is a prognostic factor for survival. HPB (Oxford) 10: 371-376, 2008.

15. Gunjan D, Rana S, Chhabra P, Parghane R, Kumar R, Dhalaria L, Mittal B and Bhasin D: Primary squamous cell carcinoma of pancreas mimicking walled-off pancreatic necrosis. J Dig Endosc 7: 17-19, 2016.

16. Ben Kridis W, Khanfir A, Toumi N, Ben Amar M, Boudawara T and Frikha M: Primary squamous cell carcinoma of the pancreas: A report of two cases and review of the literature. Intern Med 54: 1357-1359, 2015.

17. Terada T: Adenosquamous Carcinoma and Pure Squamous Cell Carcinoma of the Pancreas: Report of two Cases. Case Rep Gastroenterol 4: 369-373, 2010.

18. Tempero MA: Multidisciplinary management of pancreatic cancer. J Natl Compr Canc Netw 13 (Suppl 5): 700-702, 2015.

19. Chakraborty S and Singh S: Surgical resection improves survival in pancreatic cancer patients without vascular invasion- a population based study. Ann Gastroenterol 26: 346-352, 2013.

20. Hackert T, Sachsenmaier M, Hinz U, Schneider L, Michalski CW, Springfeld C, Strobel O, Jäger D, Ulrich A and Büchler MW: Locally advanced pancreatic cancer: Neoadjuvant therapy with folfirinox results in resectability in $60 \%$ of the patients. Ann Surg 264: 457-463, 2016.

21. Bideau K, Metges JP, Bayle S, André M, Robaszkiewicz M, Lagarde $\mathrm{N}$ and Labat JP: Treatment of squamous cell carcinoma of the pancreas with gemcitabine. Gastroenterol Clin Biol 30: 1217-1220, 2006 (In French).

22. De Souza AL and Saif MW: Squamous cell carcinoma of the pancreas. JOP 15: 630-631, 2014

23. Von Hoff DD, Ervin T, Arena FP, Chiorean EG, Infante J, Moore M, Seay T, Tjulandin SA, Ma WW, Saleh MN, et al: Increased survival in pancreatic cancer with nab-paclitaxel plus gemcitabine. N Engl J Med 369: 1691-1703, 2013. 
24. Von Hoff DD, Ramanathan RK, Borad MJ, Laheru DA, Smith LS, Wood TE, Korn RL, Desai N, Trieu V, Iglesias JL, et al: Gemcitabine plus nab-paclitaxel is an active regimen in patients with advanced pancreatic cancer: A phase I/II trial. J Clin Oncol 29: 4548-4554, 2011.

25. Kataoka K, Matsubara H, Urano F, Okamura S and Maeda M: $\mathrm{Nab}$-paclitaxel+gemcitabine therapy for adenosquamous carcinoma of the pancreas: An autopsy case. Nihon Shokakibyo Gakkai Zasshi 113: 1777-1784, 2016 (In Japanese).

26. Brijbassie A, Stelow E and Shami VM: Squamous cell carcinoma of the pancreas: A case report and review of literature. Gastroenterol Res 7: 102-104, 2014.

27. Serafini F, Rosemurgy AS II and Carey LC: Squamous cell carcinoma of the pancreas. Am J Gastroenterol 91: 2621-2622, 1996.

28. Brown HA, Dotto J, Robert M and Salem RR: Squamous cell carcinoma of the pancreas. J Clin Gastroenterol 39: 915-919, 2005 .
29. Ntanasis-Stathopoulos I, Tsilimigras DI, Georgiadou D, Kanavidis P, Riccioni O, Salla C, Psaltopoulou T and Sergentanis TN: Squamous cell carcinoma of the pancreas: A systematic review and pooled survival analysis. Eur J Cancer 79: 193-204, 2017.

30. Ahmad A, Sheikh S, Ali SM, Paithankar M, Mehta A and Nagarkar R: Nanosomal paclitaxel lipid suspension demonstrates higher response rates compared to paclitaxel in patients with metastatic breast cancer. J Cancer Sci Ther 07: 116-120, 2015.

31. Gelderblom H, Verweij J, Nooter K and Sparreboom A: Cremophor EL: The drawbacks and advantages of vehicle selection for drug formulation. Eur J Cancer 37: 1590-1598, 2001.

(7)(9) This work is licensed under a Creative Commons Attribution-NonCommercial-NoDerivatives 4.0 International (CC BY-NC-ND 4.0) License. 